\title{
Differential expression of microRNAs in decidua-derived mesenchymal stem cells from patients with pre-eclampsia
}

Guangfeng Zhao ${ }^{1}$, Xue Zhou², Shiwen Chen ${ }^{1}$, Huishuang Miao², Hongye Fan², Zhiqun Wang ${ }^{1}$, Yali Hu* and Yayi Hou ${ }^{2 *}$

\begin{abstract}
Background: Mesenchymal stem cells (MSCs) at maternal-fetal interface are considered to play an important role in the pathogenesis of pre-eclampsia (PE). microRNAs (miRNAs) also have an important influence on differentiation, maturation, and functions of MSCs. Our aim in this study was to determine the differential expression of miRNAs in decidua-derived MSCs (dMSCs) from severe PE and normal pregnancies.

Results: miRNA expression profiles in dMSCs from five patients with severe PE and five healthy pregnant women were screened using microarray. Then, bioinformatic analysis of the microarray results was performed. Out of 179 differentially expressed miRNAs, 49 miRNAs had significant $(p<0.05)$ differential expression of $\geq 2.0$-fold changes, including 21 up-regulated and 28 down-regulated. miRNA-Gene-network and miRNA-Gene ontology (GO) -network analyses were performed. Overall, 21 up-regulated and 15 down-regulated miRNAs showed high degrees in these analyses. Moreover, the significantly enriched signaling pathways and GOs were identified. The analyses revealed that pathways associated with cell proliferation, angiogenesis, and immune functions were highly regulated by the differentially expressed miRNAs, including Wnt signaling pathway, mitogen-activated protein kinase signaling pathway, transforming growth factor beta signaling pathway, T-cell receptor signaling pathway, and B cell receptor signaling pathway. Four miRNA predicted target genes, vascular endothelial growth factor A (VEGFA), indoleamine 2,3-dioxygenase, suppression of cytokine signaling 3, and serine/threonine protein phosphatase 2A $55 \mathrm{kDa}$ regulatory subunit $B$ a isoform (PPP2R2A) were all decreased in dMSCs from patients with PE. Furthermore, the physiological roles of miR-16 and miR-136 in the down-regulation of VEGFA and PPP2R2A, respectively, were confirmed through reporter assays.
\end{abstract}

Conclusions: These findings suggest that miRNAs in dMSCs may be important regulatory molecules in the development of PE.

Keywords: Pre- eclampsia, Mesenchymal stem cells, miRNAs, Microarray

\section{Background}

Pre-eclampsia (PE) affects approximately $5 \%$ of pregnancies and remains a leading cause of maternal and neonatal mortality and morbidity in the world [1]. While much research has been devoted toward this topic, the cause of PE still remains elusive [2]. Previous studies

\footnotetext{
*Correspondence: yali_hu@hotmail.com; yayihou@nju.edu.cn

'Department of Obstetrics and Gynecology, Nanjing Drum Tower Hospital,

Nanjing University Medical School, Nanjing 210008, China

${ }^{2}$ Immunology and Reproductive Biology Laboratory, Medical School \& State

Key Laboratory of Pharmaceutical Biotechnology, Nanjing University, Nanjing 210093, China
}

(c) 2014 Zhao et al.; licensee BioMed Central Ltd. This is an Open Access article distributed under the terms of the Creative Commons Attribution License (http://creativecommons.org/licenses/by/4.0), which permits unrestricted use, distribution, and reproduction in any medium, provided the original work is properly credited. The Creative Commons Public Domain Dedication waiver (http://creativecommons.org/publicdomain/zero/1.0/) applies to the data made available in this article unless otherwise stated.

indicate that the imbalanced immune system in the maternal-fetal interface may be one cause of PE [1,3,4]. Maternal immune maladaptation toward the feto-placental district is a cause for the development of defective trophoblast and related maternal-placental pathological anomalies, such as PE [5,6]. Moreover, studies have shown that $\mathrm{PE}$ results in a shift in angiogenesis and anti-angiogenic factors toward a maladaptive placental circulation $[7,8]$. It indicates that abnormality in placental vascular remodeling is also a likely pathogenesis of PE $[9,10]$. 
Mesenchymal stem cells (MSCs) are multi-potent progenitor cells, which can differentiate into various cell types, such as osteoblasts, adipocytes and chondroblasts, and are easily expanded and stored ex vivo [11,12]. MSCs are the focus of intensive efforts worldwide directed not only at elucidating their nature and unique properties but also in developing cell-based therapies for a diverse range of diseases [13]. They are considered to be immune-privileged and shown to exert a strong inhibitory effect on other immune cells [14-17]. Over 300 clinical trials related to tissue repair and immune conditions have been conducted in treatment with MSCs because of its immunosuppressive properties [18-20]. Moreover, MSCs are promising tools for treating diseases such as myocardial infarction and stroke due to their ability to promote endogenous angiogenesis and neurogenesis through a variety of secreted factors [21].

The maternal-fetal interface is an important source of MSCs [22-24]. Aberrant levels of cytokines were observed in placenta-derived MSCs from patients with PE, and higher levels of MSC negative markers were found in the placentas from patients with PE $[25,26]$. These findings suggest that MSCs may contribute to pathogenesis of PE. Therefore, investigation of the immune-modulatory, proangiogenic, and anti-inflammatory properties of deciduaderived MSCs (dMSCs) may open new perspectives into the understanding of PE [5].

MicroRNAs (miRNAs) are small noncoding RNAs that control gene expression by binding to target messenger RNAs (mRNAs) and thereby inducing translational repression or degradation of mRNAs [27]. miRNAs contribute to embryonic development and tissue homeostasis but even more profoundly regulate pathophysiological processes $[28,29]$. miRNAs also have an important influence on differentiation, maturation, and functions of stem cells [30,31]. Moreover, it was reported that differential expression of miRNAs has been observed in placentas from patients with PE $[32,33]$. Therefore, it was hypothesized that miRNAs may be involved in the pathogenesis of PE by regulating dMSCs.

In this study, for a better understanding of the pathogenesis of the PE, the miRNA expression profiles of dMSCs from patients with $\mathrm{PE}$ and healthy pregnant women were analyzed using microarray. Bioinformatic analysis of the microarray results was performed. The results showed that the differences in miRNAs and their regulated signaling pathways exist in dMSCs from healthy pregnant women and patients with PE. The expression of target genes of differentially expressed miRNAs in dMSCs from patients with PE was also detected. These findings have important implications for revealing the pathogenesis of PE.

\section{Methods}

\section{Patients and tissue samples}

Human decidua tissues from patients with PE and agematched normotensive controls were collected in the
Department of Gynecology and Obstetrics of the Affiliated Drum Tower Hospital of Nanjing University Medical School (Nanjing, China). The hospital's ethics committee approved the consent forms and the protocol for evaluating the tissue. Written consent form was received from each patient prior to surgery. PE was defined as the presence of hypertension and proteinuria beyond the 20th week of pregnancy. Elevation in blood pressure with systolic blood pressure $>140 \mathrm{~mm} \mathrm{Hg}$ or diastolic pressure $>$ $90 \mathrm{~mm} \mathrm{Hg}$ was considered hypertensive. All deciduas were obtained at the time of cesarean section. Twenty pregnant women who had complications of severe lateonset PE with delivery occurring after 34 weeks and 20 women with normal term pregnancies as the control group were recruited. The relevant clinical characteristics of the patients are presented in Table 1. Any complications of pregnancy such as multiple pregnancies including twins, fetal structural or genetic anomalies, presence of maternal chronic hypertension, hemolysis, elevated liver enzyme levels, the HELLP syndrome, cardiovascular disease, renal disease, hepatic disease, diabetes, or other infectious disease were criteria for exclusion.

\section{Isolation and culture of MSCs from deciduas}

The decidua tissues were cut into $1-2 \mathrm{~mm}^{3}$ fragments and incubated in an enzyme cocktail $(5 \mathrm{U} / \mathrm{mL}$ hyaluronidase, $125 \mathrm{U} / \mathrm{mL}$ collagenase and $50 \mathrm{U} / \mathrm{mL}$ dispase; Sigma, St Louis, MO) for 90-120 min with gentle agitation at $37^{\circ} \mathrm{C}$. This tissue was then crushed with forceps to release individual cells, and large pieces of tissue were removed. The cells were pelleted by centrifugation at $250 \mathrm{~g}$ for $5 \mathrm{~min}$, resuspended in fresh medium containing Dulbecco's modified Eagle's medium (DMEM) / F12 (Gibco, Grand Island, $\mathrm{NY}$ ) and $20 \%$ fetal bovine serum and transferred to six well plates. Cells were incubated at $37^{\circ} \mathrm{C}$ in an incubator with $5 \% \mathrm{CO}_{2}$ at saturating humidity. When cells reached $70-80 \%$ confluence or when numerous colonies were observed, the cells were detached using 0.25\% trypsin/ ethylenediaminetetraacetic acid (Invitrogen, Carlsbad, CA, USA), and the trypsin was inactivated using DMEM/F12. The culture medium was replaced every 3 or 4 days.

\section{Flow cytometry}

After passages 2-4, the specific surface antigens of dMSCs in the cultures were detected by flow cytometry analysis. The following mouse anti-human antibodies, purified or directly conjugated with fluorescein isothiocyanate, phycoerythrin, or allophycocyanin, were used in the flow cytometry analysis: anti-CD105, anti-CD73, anti-CD90, anti-CD29, anti-CD44, anti-CD106, anti-HLADR, anti-CD19, antiCD11b, anti-CD14, anti-CD34, anti-CD31, anti-CD45 and immunoglobulin (Ig) G/IgM isotype controls (all from BD Biosciences, San Jose, CA). For fluorescence measurements only, data from 10,000 single cell events were 
Table 1 Clinical characteristics of study population

\begin{tabular}{llll}
\hline & PE $(\mathbf{N}=\mathbf{2 0})$ & Control $(\mathbf{N}=\mathbf{2 0})$ & p-Value \\
\hline Age, years & $29.2 \pm 1.4$ & $28.9 \pm 1.2$ & NS \\
Gestational age at delivery, weeks & $37.3 \pm 0.3$ & $38.8 \pm 0.5$ & NS \\
\% of primiparae & $11(55 \%)$ & $14(70 \%)$ & NS \\
Body mass index, $\mathrm{kg} / \mathrm{m}^{2}$ & $28.3 \pm 0.9$ & $27.9 \pm 1.1$ & $\mathrm{NS}$ \\
Systolic blood pressure, $\mathrm{mm} \mathrm{Hg}$ & $160.4 \pm 4.7$ & $118.3 \pm 3.4$ & $<0.05$ \\
Diastolic Blood pressure, $\mathrm{mm} \mathrm{Hg}$ & $110.4 \pm 4.1$ & $82.5 \pm 3.8$ & $<0.05$ \\
Proteinuria, $\mathrm{mg} / 24 \mathrm{~h}$ & $2108 \pm 30.4$ & 0 & $<0.05$ \\
Alanine aminotransferase, $\mathrm{U} / \mathrm{L}$ & $35.3 \pm 2.3$ & $31.8 \pm 3.1$ & $\mathrm{NS}$ \\
Blood urea nitrogen, $\mathrm{mmol} / \mathrm{L}$ & $3.9 \pm 0.4$ & $3.7 \pm 0.6$ & $\mathrm{NS}$ \\
Platelet, $\times 10^{9} / \mathrm{L}$ & $160.4 \pm 32.1$ & $194.3 \pm 30.8$ & $\mathrm{NS}$ \\
Birth weight, $\mathrm{g}$ & $2901 \pm 183$ & $3278 \pm 203$ & $\mathrm{NS}$ \\
Placenta weight, $\mathrm{g}$ & $476 \pm 45$ & $513 \pm 52$ & $\mathrm{NS}$ \\
\hline
\end{tabular}

$\mathrm{PE}$, pre-eclampsia; NS, non-significant.

collected using a standard FACScalibur ${ }^{\mathrm{Tm}}$ flow cytometer (Immunocytometry Systems/Becton Dickinson, San Jose, CA). Data were analyzed using CELLQuest ${ }^{\mathrm{m}}$ (Becton Dickinson).

\section{miRNA microarray analysis, miRNA-Gene-network and} miRNA-Gene-ontology (GO) network analysis

Ten samples of dMSCs, five from women with normal pregnancies (control group, N1-N5) and five from patients with PE (P1-P5) were assayed using human miRNA microarray kit version 16.0 (Agilent Technologies, Santa Clara, CA) purchased from CapitalBio Corporation (Beijing, China). Total RNA, including miRNAs, was extracted using Trizol reagent (Invitrogen) according to the manufacturer's instructions. The concentration of RNA was measured using a SmartSpec ${ }^{\mathrm{Tm}}$ Plus spectrophotometer (Bio-Rad, Hercules, CA), and the purity of RNA was checked by Agilent 2100 Bioanalyzer (the value of A260/ A280 was between 1.9 and 2.0), and the quality of RNA was confirmed by agarose gel electrophoresis. For each miRNA, multiple probes were spotted on the array, and the mean intensity of these probes was calculated to represent the expression value of the miRNAs. In addition, multiple spots were included as negative controls. For each sample, 100 ng total RNA was hybridized with the miRNA array and further processed in accordance with the manufacturer's instructions. Only those miRNAs with significant $(\mathrm{p}<0.05)$ differential expression of $\geq 2.0$-fold changes were reported. The scanned images were processed using the Sanger Center miRBase version 16.0. The miRNA-Gene-network was constructed based on the interactions of miRNAs and genes in Sanger miRNA database. The miRNA gene ontology (GO) network was constructed based on the relationships of significant GO categories and genes/miRNAs.

\section{Quantitative reverse transcription-polymerase chain reaction analysis}

Total RNA was purified using miRNA isolation kit (Ambion, Austin, TX) to enrich the small RNA fraction. The expression of miRNAs was determined by SYBR Green assays (Bio-Rad, Hercules, CA). SYBR Green qPCR SuperMixUDG was purchased from Invitrogen. Quantitative polymerase chain reaction (qPCR) was performed using an Applied Bio- Systems 7500 Fast system. All experiments were performed in triplicate. The level of miRNA expression was calculated based on the PCR cycle number $(\mathrm{Ct})$, and the relative gene expression level was determined using the ${ }^{{ }^{\Delta}} \mathrm{Ct}$ method. All primers used are listed in Table 2.

\section{Luciferase assays}

Cells were plated in 24-well plates at a density of $1.5 \times 10^{4}$ cells per well and each well received 250 ng pGL3luciferase reporter and $5 \mathrm{ng}$ Renilla luciferase reporter. The cells were harvested using Promega's Passive Lysis buffer after the indicated treatment. Luciferase and Renilla luciferase activities were determined using Promega's Dual Luciferase assay in a Plate Chameleon luminometer (BioScan, Washington DC). Firefly luciferase was normalized by Renilla luciferase to correct for transfection efficiency. Fold induction was determined by dividing the averaged normalized values from each treatment by the control value for each transfection condition within that experiment. Values were averaged from multiple experiments as indicated in the figure legends.

\section{Data analysis}

The acquired array images were analyzed with Agilent Feature Extraction software (version 10.7.3.1). Quality normalization and subsequent data processing were performed with the Agilent GeneSpring GX software package 
Table 2 Primer information

\begin{tabular}{|c|c|c|}
\hline miRNA name & Forward primer(5'-3') & Reverse primer $\left(5^{\prime}-3^{\prime}\right)$ \\
\hline miR-136-FP & ACACTCCAGCTGGGACTCCATTTGTTTG & CTCAACTGGTGTCGTGGAGTCGGCAATTCAGTTGAGTCCATCAT \\
\hline miR-495-RP & ACACTCCAGCTGGGAAACAAACATGGTG & CTCAACTGGTGTCGTGGAGTCGGCAATTCAGTTGAGAAGAAGTG \\
\hline miR-494-RP & ACACTCCAGCTGGGTGAAACATACACGG & CTCAACTGGTGTCGTGGAGTCGGCAATTCAGTTGAGGAGGTTTC \\
\hline miR-16-RP & ACACTCCAGCTGGGTAGCAGCACGTAAA & CTCAACTGGTGTCGTGGAGTCGGCAATTCAGTTGAGCGCCAATA \\
\hline miR-29b-RP & ACACTCCAGCTGGGTAGCACCATTTTGAAA & CTCAACTGGTGTCGTGGAGTCGGCAATTCAGTTGAGAACACTGA \\
\hline miR-140-RP & ACACTCCAGCTGGGCAGTGGTITACCC & CTCAACTGGTGTCGTGGAGTCGGCAATTCAGTTGAGCTACCATA \\
\hline miR-30a-RP & ACACTCCAGCTGGGTGTAAACATCCTCG & CTCAACTGGTGTCGTGGAGTCGGCAATTCAGTTGAGCTTCCAGT \\
\hline miR-100-RP & ACACTCCAGCTGGGAACCCGTAGATCCG & CTCAACTGGTGTCGTGGAGTCGGCAATTCAGTTGAGCACAAGTT \\
\hline miR-221-RP & ACACTCCAGCTGGGAGCTACATTGTCTGC & CTCAACTGGTGTCGTGGAGTCGGCAATTCAGTTGAGGAAACCA \\
\hline miR-1207-RP & ACACTCCAGCTGGGTGGCAGGGAGGCT & CTCAACTGGTGTCGTGGAGTCGGCAATTCAGTTGAGCCCCTCCC \\
\hline U6snRNA & CTCGCTTCGGCAGCACA & AACGCTTCACGAATTTGCGT \\
\hline URP & TGGTGTCGTGGAGTCG & \\
\hline SOCS3 & CCTGCGCCTCAAGACCTTC & GTCACTGCGCTCCAGTAGAA \\
\hline PPP2R2A & CATACCAGGTGCATGAATACCTC & GGGTTATGTCTCGCTITGTGTIT \\
\hline GAPDH & GGAGCGAGATCCCTCCAAAAT & GGCTGTTGTCATACTTCTCATGG \\
\hline
\end{tabular}

(v11.5.1). Differentially expressed miRNAs were identified through Fold Change filtering and hierarchically clustered by the Agilent GeneSpring GX software (version 11.5.1). Statistical analysis was performed using unpaired Student's t-test, using Graphpad Prism 5 Demo software (GraphPad software, San Diego, CA). A p $<0.05$ was considered to be statistically significant.

\section{Results}

Identification of dMSCs derived from patients with PE and healthy donors

The decidua tissues were collected from patients with PE and age-matched normotensive controls. Then the dMSCs were isolated and cultured as described in Methods. After two cell passages, the cells formed a monolayer of homogeneous bipolar spindle-like cells with a whirlpool-like array (Figure 1A and B). Furthermore, after three cell passages, the adherent cells were symmetric with phenotypic surface antigens. The dMSCs shared most of their phenotypes with bone marrow-derived MSCs as reported previously [34-36], including positivity for CD29, CD44, CD90, CD105 (SH2) and CD73 (SH3), and negativity for CD19, CD11b, CD14, CD34, CD106, CD45 and CD31 (endothelial cell marker), and HLA-DR (Figure $1 C$ and D). The results showed that dMSCs from patients with PE and healthy controls maintained similar cell morphology and phenotype (Figure 1).

\section{miRNA expression profiles and validation of microarray data by qPCR}

Using the Agilent human miRNA microarray kit version 16.0, consisting of 940 miRNA probes, corresponding to the Sanger Center miRBase version 16.0, miRNA expression profiling was performed in the dMSCs derived from the healthy women with normal pregnancies (N1-N5) and women with PE (P1-P5). The comparison between these populations is expected to reveal some underlying differences regarding the activation of gene expression programs related to maintenance, proliferation and function of stem cells, providing important insights into the pathophysiology of PE.

To obtain an overview of the similarities and differences in their miRNA expression profiles, we performed an unsupervised clustering analysis of our expression datasets after normalizing miRNA Ct values across samples with the quantile method and filtering out miRNAs with low variation across all samples. The hierarchical cluster of the genes with $>2$-fold changes between healthy women with normal pregnancies and patients with $\mathrm{PE}$ is shown in Figure 2A. The biological replicates of each cell type were read together, demonstrating the robustness of this dataset. Remarkably, the miRNA expression profile of dMCSs from patients with PE is different from that of healthy women with normal pregnancies.

As shown in Figure 2B, compared with healthy women with normal pregnancies, miR-136, miR-494, miR-16, miR-100, miR-29b, miR-140-3p, miR-376a, miR-301a, miR-4324, miR-324-5p, miR-99a, miR-30a and miR-140$5 p$ were significantly increased, while miR-148a, miR-4270, miR-1225-5p, miR-1207, miR-638, miR-1202, miR-2861, miR-483-5p, miR-575, miR-4327, miR-4298 and miR3679-5p were decreased in dMSCs from patients with PE. Further validation of aberrant miRNAs was determined using qPCR analysis in dMSCs from 20 patients with $\mathrm{PE}$ and 20 healthy women with normal pregnancies. The nine most up-regulated miRNAs (miR-136, miR-495, miR-16, miR-29b, miR-140-5p, miR-30a, miR-100, miR-494, 


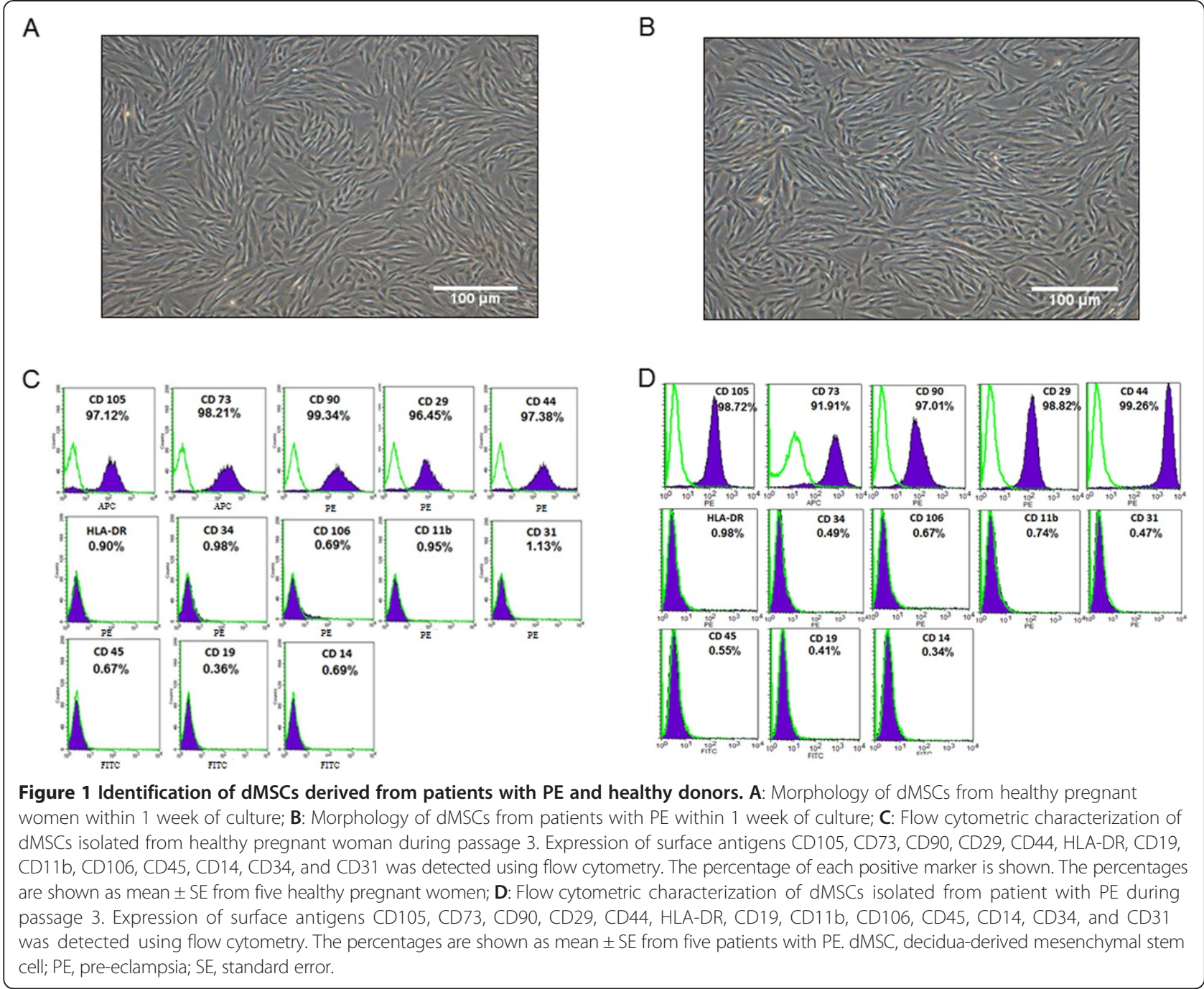

and miR-221) and one down-regulated miRNA (miR1207-5p) were identified. As shown in Figure 2C, miR136, miR-495, miR-16, miR-29b, miR-140-5p, miR-30a, miR-100, miR-494 and miR-221 were increased and miR1207-5p was decreased in dMSCs from patients with PE. The results were consistent with the microarray analysis.

Furthermore, the patients with PE were divided into more severe PE (proteinuria, $>2108 \mathrm{mg} / 24 \mathrm{~h}$, systolic blood pressure, $>160.4 \mathrm{mmHg}$; diastolic blood pressure, $>110.4 \mathrm{mmHg}$ ) and less severe PE (proteinuria, $<2108 \mathrm{mg} / 24 \mathrm{~h}$, systolic blood pressure, <160.4 mmHg; diastolic blood pressure, $<110.4 \mathrm{mmHg}$ ). Then the expression of miR-136, miR-495, miR-16, miR-29b and miR-494 was detected in these two groups using qPCR. As shown in Figure 2D, miR-136, miR-495, miR-16, miR-29b and miR-494 were expressed more in patients with more severe PE than those in patients with less severe PE. Moreover, the relationship between differentially expressed miRNAs and pathogenesis of PE was analyzed. As listed in Additional file 1: Table S1, miR-16, miR-30a, miR-29b, miR-100, miR214, miR-148a, and miR-483-5p involved the regulation of angiogenesis; miR-494, miR-140-5p, miR-16, miR-301a, miR-30a, miR-221, and miR-132 played an important role in the regulation of inflammation signaling and function of immune cells; miR-140-3p, miR-140-5p, miR-29b, miR16 , and miR-31regulated the differentiation of dMSCs.

\section{Differentially expressed miRNA-Gene-network and miRNA-GO-network analysis}

To explore the relationship between miRNAs and properties of genes, the miRNA network was constructed based on the relationship between significant GOs, genes, and miRNAs. The miRNA-Gene-network was constructed based on the interactions of miRNAs and genes in the Sanger miRNA database. The miRNA-GO-network was constructed based on the relationships between significant GO categories and genes/miRNAs. In these networks, the degree represents the contribution of an individual 


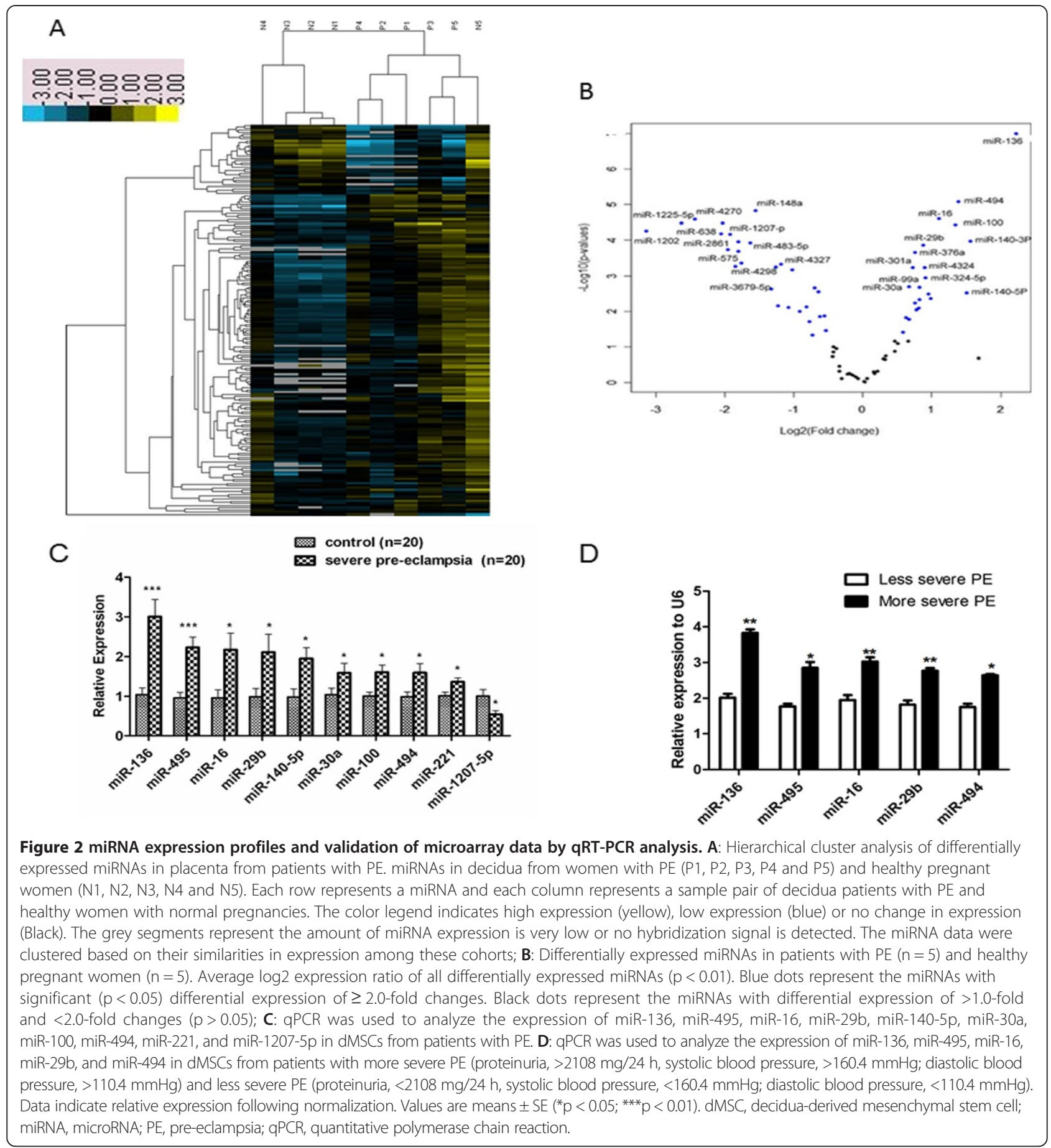

miRNA or GO category to adjacent miRNAs or GO categories.

It was reported previously [37] that miR-16 showed the highest number of connections in the up-regulated miRNAs. Other miRNAs also showed higher degrees, including miR-30a, miR-29b, miR-301a, miR-495, miR-494, miR221, miR-377, and miR-10b. In decreased miRNA-Genenetwork and miRNA-GO-network (Figure 3A and 3B), it was found that miR-1207-5p showed the highest contribution to adjacent miRNAs or GO categories in the downregulated miRNAs (Figure 3C). As shown by Additional file 1: Tables S2 and S3, miR-1207-5p regulated 30 genes and 34 GOs. Other miRNAs including miR-199b-5p (24 genes and 28 GOs), miR-940 (23 genes and 21 GOs), miR-148a (21 genes and 21 GOs) and miR-214 (20 genes and 28 GOs) also showed a higher number 
of connections with adjacent miRNAs or GO categories (Figure 3C, Additional file 1: Tables S2 and S3).

\section{Analysis of the signaling pathways regulated by} differentially expressed miRNAs

Furthermore, the significantly enriched signaling pathways, which regulated by the differentially expressed miRNAs, were identified. The top 15 enriched signaling pathways for up-regulated and down-regulated expression of miRNAs were shown in Additional file 1: Figure S1A and $\mathrm{S} 1 \mathrm{~B}$, respectively. It revealed that the pathways associated with cell proliferation, angiogenesis, and immune functions were highly regulated by differentially expressed miRNAs, including Wnt signaling pathway, mitogenactivated protein kinase (MAPK) signaling pathway, transforming growth factor (TGF) beta signaling pathway, $\mathrm{T}$ cell receptor signaling pathway, and B cell receptor signaling pathway, etc. (Additional file 1: Figure S1A and S1B). Moreover, the significantly enriched GOs regulated by differentially expressed miRNAs were analyzed. The degree represents the number of miRNAs that regulate the same GO. As shown in Table 3, angiogenesis, response to hypoxia, apoptosis, TGF beta receptor signaling pathway, cell migration, and immune response

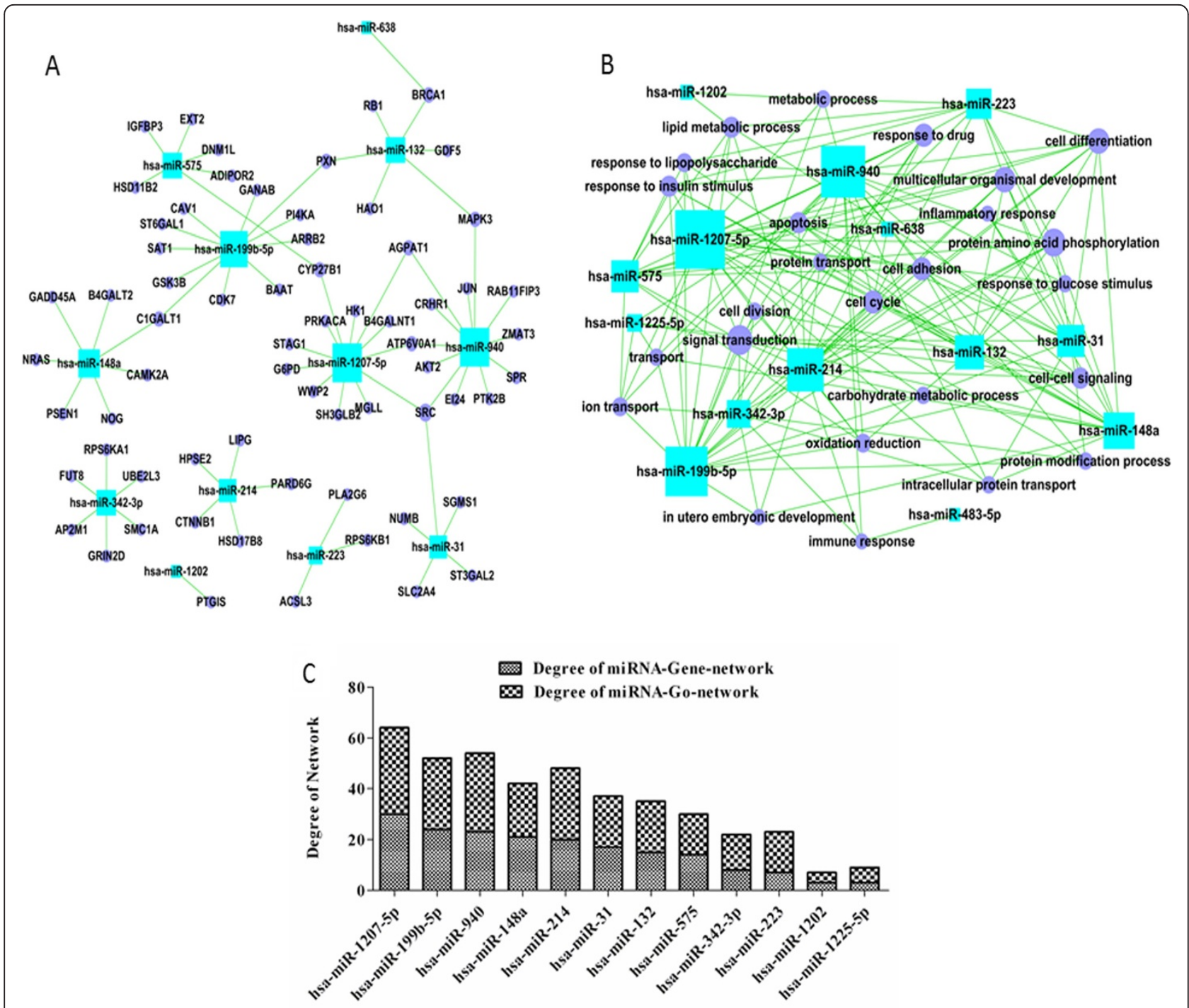

Figure 3 Differentially expressed miRNA-Gene-network and miRNA-GO-network analysis. A: The miRNA-Gene-network was constructed based on the interactions of down-regulated miRNAs and genes in Sanger miRNA database; B: The miRNA-GO-network was constructed based on the relationships of significant GO categories and down-regulated miRNAs; $\mathbf{C}$ : The network degree of low-level expressed miRNAs. In these networks, the term "degree" represents the contribution of an individual miRNA or GO category to adjacent genes or GO categories. GO, gene ontology; miRNA, microRNA. 
were significantly regulated by up-regulated miRNAs in patients with PE. In these GOs, angiogenesis and response to hypoxia were regulated by 15 miRNAs and 14 miRNAs, respectively. Moreover, cell differentiation, cell cycle, apoptosis, aging, and response to lipopolysaccharide were mainly regulated by down-regulated miRNAs in patients with PE (Table 3).

\section{Detection of VEGFA, IDO, SOCS3 and PPP2R2A expression in dMSCs}

Vascular endothelial growth factor A (VEGFA) is a key angiogenesis factor. Indoleamine 2,3-dioxygenase (IDO) and suppression of cytokine signaling 3 (SOCS3) play an important role in immunoloregulation of MSC. Serine/ threonine protein phosphatase $2 \mathrm{~A} 55 \mathrm{kDa}$ regulatory subunit $\mathrm{B} \alpha$ isoform (PPP2R2A) negatively regulates extracellular signal-regulated kinase (ERK) pathway [38,39], which is involved in differentiation of MSCs $[40,41]$.

Interestingly, VEGFA was predicted to be a putative target of miR-16 and miR-29b by the miRNA-Gene-network analysis and other target prediction programs [miRanda (http://diana.cslab.ece.ntua.gr/microT/), TargetScan (http:// www.targetscan.org/) and PicTar (http://pictar.mdc-berlin. $\mathrm{de} /$ ) algorithms). With same methods, it was predicted that IDO was a target of miR-494 and SOCS3 was a target of miR-495. In addition, it was reported that PPP2R2A is the target gene of miR-136 [38]. To test whether these changes in miRNA expression were accompanied by changes in corresponding genes, the expression levels of VEGFA, IDO, SOCS3, and PPP2R2A in dMSCs were determined. As shown in Figure 4A-4D, VEGFA, IDO, SOCS3 and PPP2R2A were all decreased in dMSCs from patients with $\mathrm{PE}$ compared with healthy pregnant women.

Table 3 Target gene signaling pathways analysis

\begin{tabular}{ll}
\hline go_name & Degree \\
\hline Increased miRNAs in patients with PE & 15 \\
Angiogenesis & 14 \\
Response to hypoxia & 13 \\
Apoptosis & 12 \\
Transforming growth factor beta receptor signaling pathway & 11 \\
Cell migration & 11 \\
Immune response & \\
\hline Decreased miRNAs in patients with PE & 9 \\
Cell differentiation & 8 \\
Cell cycle & 7 \\
Apoptosis & 6 \\
Aging & 6 \\
Response to lipopolysaccharide &
\end{tabular}

Degree represents the number of miRNAs related to a GO.
miR-16 targets VEGFA and miR-136 targets PPP2R2A in dMSCs

miR-16 showed the highest number of connections in the differentially expressed miRNAs. More importantly, miR-16 was predicted to target VEGFA, which is an important candidate for the pathogenesis of PE. In addition, miR-136 was found to be highly up-regulated in the validation experiment. Therefore, miR-16 and miR-136 were chosen to validate the predicted target gene in dMSCs. The expression of a luciferase reporter gene fused to 3' untranslated region (UTR) of VEGFA after transfection with miR-16 mimic and its inhibitor into dMSCs was detected. The results showed that miR-16 significantly suppressed the activity of luciferase, which could be reversed by further introduction of miR-16 inhibitor in dMSCs (Figure 5A). Similarly, miR-136 significantly suppressed the expression of a luciferase reporter gene fused to 3 ' UTR of PPP2R2A, which could be reversed by further introduction of miR-136 inhibitor in dMSCs (Figure 5B). These results further indicate that differentially expressed miRNAs may be involved in the pathogenesis of PE.

\section{Discussion}

Previous studies indicated that PE may be a pregnancyinduced autoimmune disease, and the imbalanced immune system in the maternal-fetal interface may be one cause of PE $[1,3,4]$. Moreover, abnormality in placental vascular remodeling is also a likely pathogenesis of $\mathrm{PE}$ $[9,10]$. These are the two main theories for explaining the development of PE [42]. MSCs are considered to be immune-privileged and shown to exert a strong inhibitory effect on other immune cells [14-17], and they have the ability to promote endogenous angiogenesis and neurogenesis through a variety of secreted factors [21]. The maternal-fetal interface is an important source of MSCs [22-24]. Therefore, investigation of immune-modulatory, pro-angiogenic and anti-inflammatory properties of MSCs at the maternal-fetal interface may open new perspectives into the understanding of PE [5].

In the present study, miRNA expression profiles showed differentially expressed miRNAs in dMSCs between healthy pregnant women and patients with PE. Differential miRNA expressions were defined as a statistically significant difference with a $\geq 2$-fold change. Significantly up-regulated 21 miRNAs and down-regulated 28 miRNAs are present in patients with PE vs healthy pregnant women. Nine top upregulated miRNAs including miR-136, miR-495, miR-16, miR-29b, miR-140-5p, miR-30a, miR-100, miR-494, and miR-221 and one down-regulated miRNA, miR-1207-5p, were confirmed by qPCR. A previous study had shown that miR-16 inhibits the proliferation and angiogenesisregulating potential of $\mathrm{dMSC}$ [37]. miR-29b contributes to PE through its effects on apoptosis, invasion and angiogenesis of trophoblast cells [43]. Moreover, another up- 


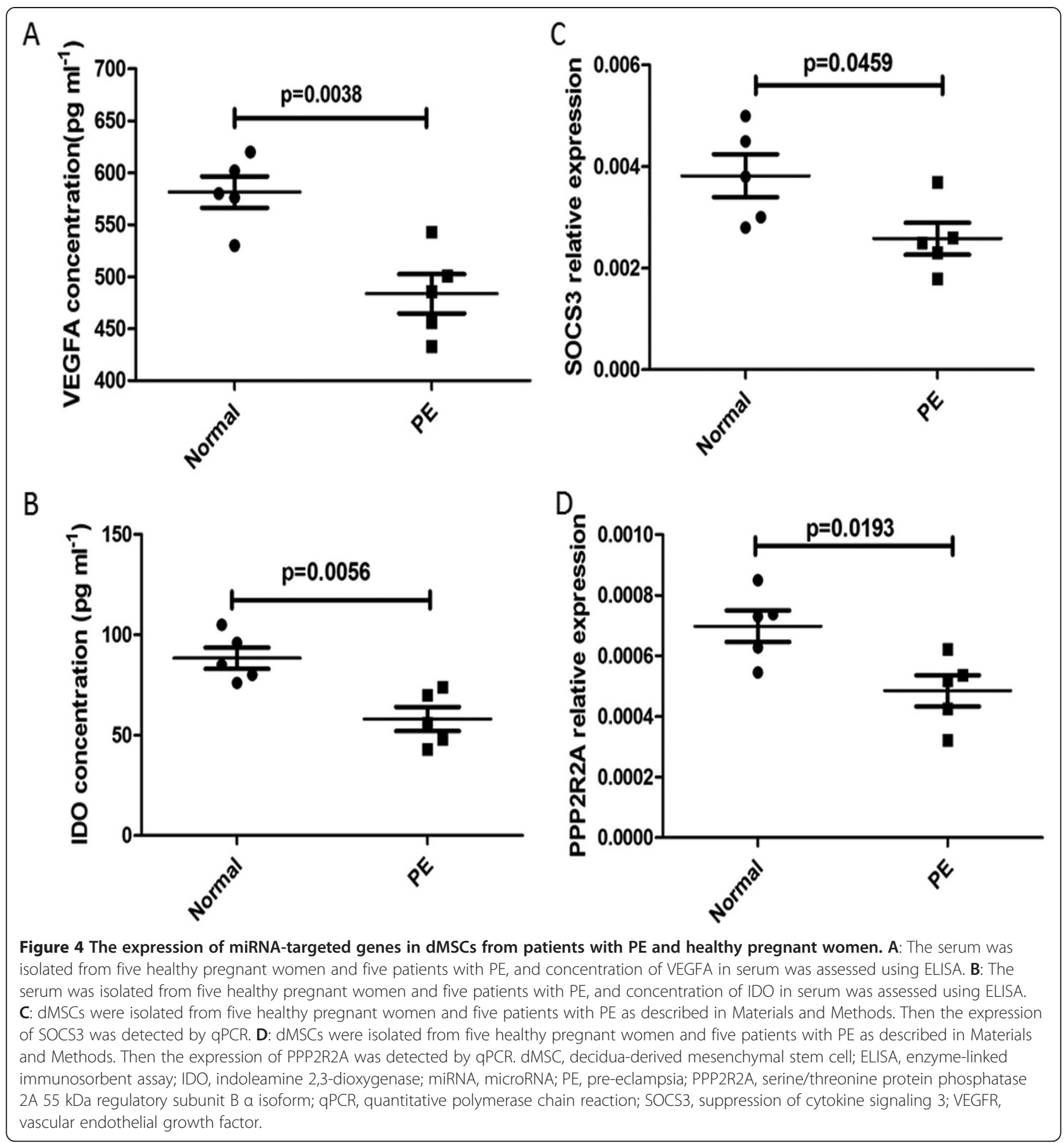

regulated miRNA, miR-181a, was proved to regulate immune balance by inhibiting proliferation and immunosuppressive properties of MSCs [44]. Interestingly, it was found that the levels of miR-136, miR-495, miR-16, miR-29b and miR-494 were more in patients with more severe $\mathrm{PE}$ (proteinuria, >2108 mg/24 h; systolic blood pressure, >160.4 mm Hg; diastolic blood pressure, $>110.4 \mathrm{~mm}$ $\mathrm{Hg}$ ) than those in patients with less severe PE (proteinuria,
<2108 mg/24 h; systolic blood pressure, <160.4 mm Hg; diastolic blood pressure, $<110.4 \mathrm{~mm} \mathrm{Hg}$ ).

Target gene signaling pathway analysis showed that angiogenesis and response to hypoxia were significantly regulated by differentially expressed miRNAs. Abnormality in placental vascular remodeling is a likely pathogenesis of PE $[9,10]$. In particular, an imbalance in circulating proangiogenic and antiangiogenic factors released by the 

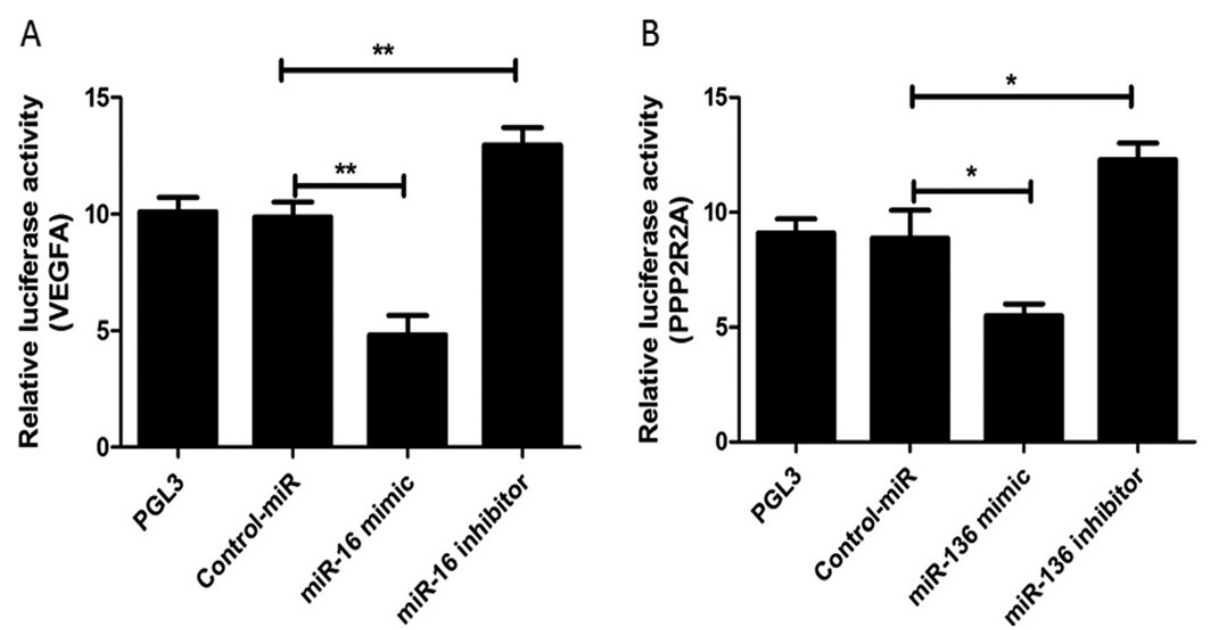

Figure 5 Validation of miRNA target. A: The luciferase activity of dMSCs was measured after co-transfection with the indicated VEGFA $3^{\prime}$ UTR constructs and miR-16 or its inhibitor for $24 \mathrm{~h}$. B: The luciferase activity of dMSCs was measured after co-transfection with the indicated PPP2R2A $3^{\prime}$ UTR constructs and miR-136 or its inhibitor for $24 \mathrm{~h}$. The results are shown as mean \pm SE from three representative independent experiments. ${ }^{*} p<0.05$, ${ }^{*} P<0.01$ compared with miR-negative. dMSC, decidua-derived mesenchymal stem cell; miRNA, microRNA; PPP2R2A, serine/threonine protein phosphatase 2A $55 \mathrm{kDa}$ regulatory subunit B a isoform; SE, standard error, UTR, untranslated region; VEGFR, vascular endothelial growth factor.

hypoxic placenta has gained currency as a critical link between placental dysfunction and several maternal manifestations of PE, particularly endothelial dysfunction and proteinuria [45]. Besides, it was reported that miR-16 and miR-29b, which could regulate angiogenesis $[37,43]$, and another three up-regulated miRNAs, miR-140, miR-30a, and miR-100, were also associated with angiogenesis [46-48].

GO analysis also showed that miRNAs involve many immune response signaling pathways in patients with $\mathrm{PE}$ including the MAPK signaling pathway, TGF beta signaling pathway, T-cell receptor signaling pathway, and B-cell receptor signaling pathway, etc. In a previous study, it was found that miR-181a is an immune-regulating factor [44]. miR-181a can regulate the TGF-beta signaling pathway by targeting TGFBR1 and TGFBRAP1 in dMSCs. In addition, miR-181a can also enhance secretion of interleukin (IL)-6 and IDO by activating p38 and JNK signaling pathways in dMSCs [44]. Moreover, it was reported that miR-16 modulates nuclear factor-kappaB-regulated transactivation of IL-8 gene [49]. miRNA-29 is involved in the adaptive immune system and immune-modulation [50,51]. miR-140 regulates TGF- $\beta 1 /$ Smad3 pathway [52]. miRNA30a and miRNA-221 regulate B cells and mast cells, respectively $[53,54]$.

\section{Conclusions}

miRNA expression profiles of patients with PE are significantly different from that of healthy pregnant women. Altered miRNAs lead to excessive activation or inactivation of signaling pathways in dMSCs. These aberrant changes result in abnormality of immune-modulatory, pro-angiogenic and anti-inflammatory properties of dMSCs. Our study further indicates that differentially expressed miRNAs may be involved in the pathogenesis of PE.

\section{Additional file}

Additional file 1: Figure S1. Target gene signaling pathways analysis. A. The significantly enriched signaling pathways which regulated by up-regulated miRNAs. The top fifteen enriched signaling pathways were showed; B. The significantly enriched signaling pathways which regulated by down-regulated miRNAs. The top fifteen enriched signaling pathways were showed. Table S1. The functions of differential expressed miRNAs. Table S2. Decreased miRNA-Gene-network. Table S3. Decreased miRNA-GO-network.

\section{Abbreviations}

MSC: Mesenchymal stem cell; dMSC: Decidua-derived mesenchymal stem cell; PE: Pre-eclampsia; miRNAs: microRNAs; TGF beta: Transforming growth factor beta; MAPK: Mitogen-activated protein kinase.

\section{Competing interests}

The authors declared that they have no competing interests.

\section{Authors' contributions}

ZGF, HYY and HYL designed and carried out the experiments and wrote the manuscript. ZX, CSW, MHS, FHY and WZQ discussed the experimental design and results with ZGF and participated in manuscript writing. All authors read and approve the final manuscript.

\section{Acknowledgments}

This work was supported by the Natural Science Funds of Jiangsu Province (BK20130091), National Natural Science Foundation of China (H0420), and China Postdoctoral Science Foundation funded project (2012 M521063).

Received: 7 February 2014 Accepted: 12 August 2014

Published: 19 August 2014 


\section{References}

1. Zhou CC, Zhang YJ, Irani RA: Angiotensin receptor agonistic autoantibodies induce pre-eclampsia in pregnant mice. Nat Med 2008, 14:855-862.

2. Redman CW, Sargent IL: Latest advances in understanding pre-eclampsia. Science 2005, 308:1592-1594.

3. Kanasaki K, Kalluri R: The biology of preeclampsia. Kidney Int 2009, 76:831-837.

4. Goldman-Wohl DS, Yagel S: Examination of distinct fetal and maternal molecular pathways suggests a mechanism for the development of preeclampsia. J Reprod Immunol 2007, 76:54-60.

5. Rolfo A, Giuffrida D, Nuzzo AM: Pro-inflammatory profile of preeclamptic placental mesenchymal stromal cells: new insights into the etiopathogenesis of preeclampsia. PLoS One 2013, 8(3):e59403.

6. Erlebacher A: Immunology of the maternal-fetal interface. Annu Rev Immunol 2013, 31:387-411.

7. Venkatesha S, Toporsian M, Lam C, Hanai J, Mammoto T, Kim YM: Soluble endoglin contributes to the pathogenesis of preeclampsia. Nat Med 2006, 12:642-649

8. Verlohren I, Stepan H, Dechend R: Angiogenic growth factors in the diagnosis and prediction of pre-eclampsia. Clin Sci 2012, 122:43-52.

9. Pijnenborg R, Vercruysse L, Hanssens M: The uterine spiral arteries in human pregnancy: facts and controversies. Placenta 2006, 7:939-958.

10. Perni EU, Sison C, Sharma V, Helseth G, Hawfield A, Suthanthiran M: Angiogenic Factors in Superimposed Preeclampsia A Longitudinal Study of Women With Chronic Hypertension During Pregnancy. Hypertension 2012, 59:740-746.

11. Karahuseyinoglu S, Cinar O, Kilic E: Biology of stem cells in human umbilical cord stroma: in situ and in vitro surveys. Stem Cells 2007, 25:319-331.

12. Liu L, Zhao X, Li P, Zhao G, Wang Y, Hou Y: A novel way to isolate MSCs from umbilical cords. Eur J Immunol 2012, 42:2190-2193.

13. Bianco P, Cao X, Frenette PS, Mao JJ, Robey PG, Simmons PJ: The meaning, the sense and the significance: translating the science of mesenchymal stem cells into medicine. Nat Med 2013, 19(1):35-42

14. Crop MJ, Baan CC, Korevaar SS: Human adipose tissue-derived mesenchymal stem cells induce explosive T-cell proliferation. Stem Cells Dev 2010, 19:1843-1853.

15. Asari S, Itakura S, Ferreri K: Mesenchymal stem cells suppress B-cell terminal differentiation. Exp Hematol 2009, 37:604-615.

16. Giuliani M, Oudrhiri N, Noman ZM: Human mesenchymal stem cells derived from induced pluripotent stem cells down-regulate NK-cell cytolytic machinery. Blood 2011, 118:3254-3262.

17. Spaggiari GM, Abdelrazik H, Becchetti F: MSCs inhibit monocyte-derived DC maturation and function by selectively interfering with the generation of immature DCs: central role of MSC-derived prostaglandin E2. Blood 2009, 113:6576-6583.

18. Zhang Q, Shi S, Liu Y: Mesenchymal stem cells derived from human gingiva are capable of immunomodulatory functions and ameliorate inflammation-related tissue destruction in experimental colitis. J Immunol 2009, 183:7787-7798.

19. Sun L, Wang $D$, Liang J: Umbilical cord mesenchymal stem cell transplantation in severe and refractory systemic lupus erythematosus. Arthritis Rheum 2010, 62:2467-2475.

20. Sun L, Akiyama K, Zhang H: Mesenchymal stem cell transplantation reverses multiorgan dysfunction in systemic lupus erythematosus mice and humans. Stem Cell 2009, 27:1421-1432.

21. Hsieh JY, Wang HW, Chang SJ, Liao KH, Lee IH, Lin WS: Mesenchymal stem cells from human umbilical cord express preferentially secreted factors related to neuroprotection, neurogenesis, and angiogenesis. PLoS One 2013, 8(8):e72604

22. Cutler AJ, Limbani V, Girdlestone J: Umbilical cord-derived mesenchymal stromal cells modulate monocyte function to suppress $\mathrm{T}$ cell proliferation $\mathrm{J}$ Immunol 2010, 185:6617-6623.

23. Atkinson K, Chatterjee K, Barlow S: Potential for mesenchymal stem cell isolation from human placenta. Blood 2005, 106:158b-158b.

24. Schwab KE, Gargett CE: Co-expression of two perivascular cell markers isolates mesenchymal stem-like cells from human endometrium. Hum Reprod 2007, 22:2903-2911.

25. Hwang JH, Lee MJ, Seok OS: Cytokine expression in placentaderived mesenchymal stem cells in patients with pre-eclampsia and normal pregnancies. Cytokine 2010, 49:95-101.
26. Benian A, Uzun H, Aydin S: Placental stem cell markers in preeclampsia. Int J Gynaecol Obstet 2008, 100:228-233.

27. Bartel DP: MicroRNAs: genomics, biogenesis, mechanism, and function. Cell 2004, 116:281-297.

28. Small EM, Olson EN: Pervasive roles of microRNAs in cardiovascular biology. Nature 2011, 469:336-342.

29. Di Leva G, Calin GA, Croce CM: MicroRNAs: fundamental facts and involvement in human diseases. Birth Defects Res C Embryo Today 2006, 78:180-189.

30. Heinrich EM, Dimmeler S: MicroRNAs and stem cells: control of pluripotency, reprogramming, and lineage commitment. Circ Res 2012, 110:1014-1022.

31. Leonardo TR, Schultheisz HL, Loring JF, Laurent LC: The functions of microRNAs in pluripotency and reprogramming. Nat Cell Biol 2012, 14:1114-1121

32. Noack F, Ribbat-Idel J, Thorns C, Chiriac A, Axt-Fliedner R, Diedrich K: miRNA expression profiling in formalin-fixed and paraffin-embedded placental tissue samples from pregnancies with severe preeclampsia. J Perinat Med 2011, 39:267-271.

33. Hu Y, Li P, Hao S, Liu L, Zhao J, Hou Y: Differential expression of microRNAs in the placentae of Chinese patients with severe preeclampsia. Clin Chem Lab Med 2009, 47:923-929.

34. Castrechini NM, Murthi P, Qin S, Kusuma GD, Wilton L, Abumaree M, Gronthos S, Zannettino A, Gude NM, Brennecke SP, Kalionis B: Decidua parietalis-derived mesenchymal stromal cells reside in a vascular niche within the choriodecidua. Reprod Sci 2012, 19(12):1302-1314.

35. Kanematsu D, Shofuda T, Yamamoto A, Ban C, Ueda T, Yamasaki M, Kanemura Y: Isolation and cellular properties of mesenchymal cells derived from the decidua of human term placenta. Differentiation 2011, 82(2):77-88.

36. Hass R, Kasper C, Böhm S, Jacobs R: Different populations and sources of human mesenchymal stem cells (MSC): A comparison of adult and neonatal tissue-derived MSC. Cell Commun Signal 2011, 9:12.

37. Wang Y, Fan H, Zhao G, Liu D, Du L, Wang Z, Hu Y, Hou Y: miR-16 inhibits the proliferation and angiogenesis-regulating potential of mesenchymal stem cells in severe pre-eclampsia. FEBS J 2012, 279(24):4510-4524

38. Cheng Y, Liu W, Kim ST, Sun J, Lu L, Sun J, Zheng SL, Isaacs WB, Xu J: Evaluation of PPP2R2A as a prostate cancer susceptibility gene: a comprehensive germline and somatic study. Cancer Genet 2011, 204(7):375-381.

39. Zhou C, Zhang $X, X u L$, Wu T, Cui L, Xu D: Taurine promotes human mesenchymal stem cells to differentiate into osteoblast through the ERK pathway. Amino Acids 2014, [Epub ahead of print].

40. Xu D, Xu L, Zhou C, Lee WY, Wu T, Cui L, Li G: Salvianolic acid B promotes osteogenesis of human mesenchymal stem cells through activating ERK signaling pathway. Int J Biochem Cell Biol 2011, 51C:1-9.

41. Roberts JM, Hubel CA: The two stage model of preeclampsia: variations on the theme. Placenta 2009, 30:S32-S37.

42. Shen S, Yue H, Li Y, Qin J, Li K, Liu Y, Wang J: Upregulation of miR-136 in human non-small cell lung cancer cells promotes Erk $1 / 2$ activation by targeting PPP2R2A. Tumour Biol 2014, 35(1):631-640.

43. Li P, Guo W, Du L, Zhao J, Wang Y, Liu L, Hu Y, Hou Y: microRNA-29b contributes to pre-eclampsia through its effects on apoptosis, invasion and angiogenesis of trophoblast cells. Clin Sci (Lond) 2013, 124(1):27-40.

44. Liu L, Wang Y, Fan H, Zhao X, Liu D, Hu Y, Kidd AR 3rd, Bao J, Hou Y MicroRNA-181a regulates local immune balance by inhibiting proliferation and immunosuppressive properties of mesenchymal stem cells. Stem Cells 2012, 30(8):1756-1770.

45. Maynard SE, Karumanchi SA: Angiogenic factors and preeclampsia. Semin Nephrol 2011, 31:33-46.

46. Izzotti A, Calin GA, Arrigo P, Steele VE, Croce CM, De Flora S: Downregulation of microRNA expression in the lungs of rats exposed to cigarette smoke. FASEB J 2009, 23(3):806-812.

47. Huang QB, Ma X, Zhang X, Liu SW, Ai Q, Shi TP, Zhang Y, Gao Y, Fan Y, Ni D, Wang BJ, Li HZ, Zheng T: Down-Regulated miR-30a in Clear Cell Renal Cell Carcinoma Correlated with Tumor Hematogenous Metastasis by Targeting Angiogenesis-Specific DLL4. PLoS One 2013, 8(6):e67294.

48. Grundmann S, Hans FP, Kinniry S, Heinke J, Helbing T, Bluhm F, Sluijter JP, Hoefer I, Pasterkamp G, Bode C, Moser M: MicroRNA-100 regulates neovascularization by suppression of mammalian target of rapamycin in 
endothelial and vascular smooth muscle cells. Circulation 2011, 123(9):999-1009.

49. Zhou R, Li X, Hu G, Gong AY, Drescher KM, Chen XM: miR-16 targets transcriptional corepressor SMRT and modulates NF-kappaB-regulated transactivation of interleukin-8 gene. PLoS One 2012, 7(1):e30772.

50. Liston A, Papadopoulou AS, Danso-Abeam D, Dooley J: MicroRNA-29 in the adaptive immune system: setting the threshold. Cell Mol Life Sci 2012, 69(21):3533-3541.

51. Schmitt MJ, Margue C, Behrmann I, Kreis S: MiRNA-29: a microRNA family with tumor-suppressing and immune-modulating properties. Curr Mol Med 2013, 13(4):572-585

52. Wang C, Song X, Li Y, Han F, Gao S, Wang X, Xie S, Lv C: Low-Dose Paclitaxel Ameliorates Pulmonary Fibrosis by Suppressing TGF- $\beta 1 / S m a d 3$ Pathway via miR-140 Upregulation. PLoS One 2013, 8(8):e70725.

53. Liu Y, Dong J, Mu R, Gao Y, Tan X, Li Y, Li Z, Yang G: MicroRNA-30a promotes $B$ cell hyperactivity in patients with systemic lupus erythematosus by direct interaction with Lyn. Arthritis Rheum 2013, 65(6):1603-1611.

54. Mayoral RJ, Pipkin ME, Pachkov M, van Nimwegen E, Rao A, Monticelli S: MicroRNA-221-222 regulate the cell cycle in mast cells. J Immunol 2009, 182(1):433-445.

doi:10.1186/s12929-014-0081-3

Cite this article as: Zhao et al:: Differential expression of microRNAs in decidua-derived mesenchymal stem cells from patients with pre-eclampsia. Journal of Biomedical Science 2014 21:81.

\section{Submit your next manuscript to BioMed Central and take full advantage of:}

- Convenient online submission

- Thorough peer review

- No space constraints or color figure charges

- Immediate publication on acceptance

- Inclusion in PubMed, CAS, Scopus and Google Scholar

- Research which is freely available for redistribution 